\title{
Local Fractional Function Decomposition Method for Solving Inhomogeneous Wave Equations with Local Fractional Derivative
}

\author{
Shun-Qin Wang, ${ }^{1}$ Yong-Ju Yang, ${ }^{1}$ and Hassan Kamil Jassim ${ }^{2}$ \\ ${ }^{1}$ School of Mathematics and Statistics, Nanyang Normal University, Nanyang 473061, China \\ ${ }^{2}$ Department of Mathematics, University of Mazandaran, Babolsar 47416-95447, Iran \\ Correspondence should be addressed to Yong-Ju Yang; tomjohn1007@126.com
}

Received 17 November 2013; Accepted 9 December 2013; Published 2 January 2014

Academic Editor: Hossein Jafari

Copyright (C) 2014 Shun-Qin Wang et al. This is an open access article distributed under the Creative Commons Attribution License, which permits unrestricted use, distribution, and reproduction in any medium, provided the original work is properly cited.

\begin{abstract}
We propose the local fractional function decomposition method, which is derived from the coupling method of local fractional Fourier series and Yang-Laplace transform. The forms of solutions for local fractional differential equations are established. Some examples for inhomogeneous wave equations are given to show the accuracy and efficiency of the presented technique.
\end{abstract}

\section{Introduction}

Fractional differential equations with arbitrary orders [1] have attracted more and more attention to their extensive applications in various areas, such as physics, applied mathematics, and biology [2-8]. As a result, great deal of methods for solving the fractional differential equations are developed [9-21], such as the heat balance integral method $[9,10]$, the homotopy analysis method [11], the variational iteration method [12], the homotopy decomposition method [13, 14], and the Adomian decomposition method $[15,16]$.

The fractional differential equations were considered in sense of the Caputo derivative, the Riemann-Liouville derivative, and the Grünwald-Letnikov derivative [17]. However, they do not deal with the nondifferentiable functions defined on Cantor sets. Local fractional derivative $[18,19]$ is the best method for describing the nondifferential problems defined on Cantor sets. For example, the heat equations arising in fractal transient conduction were investigated in [19-22]. The Helmholtz and diffusion equations on the Cantor sets within local fractional derivative were discussed [23]. The NavierStokes equations on Cantor sets were suggested in [24]. There are some methods for solving the local fractional differential equations, such as the local fractional variational iteration method [20], the Yang-Fourier transform [21], the YangLaplace transform [22], the local fractional Fourier series method [25], and the local fractional Adomian decomposition method [26].

In this paper, our aims are to present the coupling method of local fractional series method and Yang-Laplace transform, which is called as the local fractional function decomposition method, and to use it to solve the differential equations with local fractional derivative. The organization of the manuscript is as follows. In Section 2, the basic mathematical tools are introduced. In Section 3, the local fractional function decomposition method for solving the differential equations with local fractional derivative is investigated. In Section 4, several examples are considered. Finally, in Section 5 the conclusions are given.

\section{Mathematical Fundamentals}

In this section, we introduce the basic notions of local fractional continuity, local fractional derivative, local fractional Fourier series, and special function in fractal space $[18,19]$, which are used in the paper.

Definition 1. Suppose that there is [19]

$$
\left|f(x)-f\left(x_{0}\right)\right|<\varepsilon^{\alpha}
$$


with $\left|x-x_{0}\right|<\delta$, for $\varepsilon, \delta>0$ and $\varepsilon, \delta \in R$; then $f(x)$ is called local fractional continuous at $x=x_{0}$ and it is denoted by $\lim _{x \rightarrow x_{0}} f(x)=f\left(x_{0}\right)$.

Definition 2. Suppose that the function $f(x)$ satisfies condition (1), for $x \in(a, b)$; it is so-called local fractional continuous on the interval $(a, b)$, denoted by

$$
f(x) \in C_{\alpha}(a, b) .
$$

Definition 3. In fractal space, let $f(x) \in C_{\alpha}(a, b)$, local fractional derivative of $f(x)$ of order $\alpha$ at $x=x_{0}$ is given by [19]

$$
\begin{aligned}
D_{x}^{(\alpha)} f\left(x_{0}\right) & =f^{(\alpha)}\left(x_{0}\right)=\left.\frac{d^{\alpha} f(x)}{d x^{\alpha}}\right|_{x=x_{0}} \\
& =\lim _{x \rightarrow x_{0}} \frac{\Delta^{\alpha}\left(f(x)-f\left(x_{0}\right)\right)}{\left(x-x_{0}\right)^{\alpha}},
\end{aligned}
$$

where $\Delta^{\alpha}\left(f(x)-f\left(x_{0}\right)\right) \cong \Gamma(1+\alpha) \Delta\left(f(x)-f\left(x_{0}\right)\right)$.

Local fractional derivative of high order and local fractional partial derivative are defined, respectively, in the following forms $[18,19]$ :

$$
\begin{aligned}
f^{(k \alpha)}(x) & =\overbrace{D_{x}^{(\alpha)} \cdots D_{x}^{(\alpha)}}^{k \text { times }} f(x), \\
\frac{\partial^{k \alpha}}{\partial x^{k \alpha}} f(x, y) & =\overbrace{\frac{\partial^{\alpha}}{\partial x^{\alpha}} \cdots \frac{\partial^{\alpha}}{\partial x^{\alpha}}}^{k \text { times }} f(x, y) .
\end{aligned}
$$

Definition 4. In fractal space, the Mittage Leffler function, sine function, cosine function, hyperbolic sine function, and hyperbolic cosine function are, respectively, defined by $[18$, 19]

$$
\begin{gathered}
E_{\alpha}\left(x^{\alpha}\right):=\sum_{K=0}^{\infty} \frac{x^{\alpha k}}{\Gamma(1+k \alpha)}, \quad x \in R, 0<\alpha \leq 1, \\
\sin _{\alpha} x^{\alpha}:=\sum_{K=0}^{\infty}(-1)^{k} \frac{x^{\alpha(2 k+1)}}{\Gamma[1+(2 k+1) \alpha]}, \quad x \in R, 0<\alpha \leq 1, \\
\cos _{\alpha} x^{\alpha}:=\sum_{K=0}^{\infty}(-1)^{k} \frac{x^{2 \alpha k}}{\Gamma(1+2 k \alpha)}, \quad x \in R, 0<\alpha \leq 1, \\
\sin h_{\alpha} x^{\alpha}:=\frac{E_{\alpha}\left(x^{\alpha}\right)+E_{\alpha}\left(-x^{\alpha}\right)}{2}, \quad x \in R, 0<\alpha \leq 1, \\
\cos h_{\alpha} x^{\alpha}:=\frac{E_{\alpha}\left(x^{\alpha}\right)-E_{\alpha}\left(-x^{\alpha}\right)}{2}, \quad x \in R, 0<\alpha \leq 1 .
\end{gathered}
$$

Definition 5. Let $f(x)$ be $2 l$-periodic. For $k \in Z$ and $f(x) \in$ $C_{\alpha}(-\infty,+\infty)$, the local fraction Fourier series of $f(x)$ is defined as (see $[18,25])$

$$
f(x)=\frac{a_{0}}{2}+\sum_{k=1}^{\infty}\left(a_{k} \cos _{\alpha} \frac{\pi^{\alpha}(k x)^{\alpha}}{l^{\alpha}}+b_{k} \sin _{\alpha} \frac{\pi^{\alpha}(k x)^{\alpha}}{l^{\alpha}}\right),
$$

where

$$
\begin{aligned}
& a_{k}=\frac{1}{l^{\alpha}} \int_{-l}^{+l} f(x) \cos _{\alpha} \frac{\pi^{\alpha}(k x)^{\alpha}}{l^{\alpha}}(d x)^{\alpha}, \\
& b_{k}=\frac{1}{l^{\alpha}} \int_{-l}^{+l} f(x) \sin _{\alpha} \frac{\pi^{\alpha}(k x)^{\alpha}}{l^{\alpha}}(d x)^{\alpha}
\end{aligned}
$$

are the local fraction Fourier coefficients.

Definition 6. Let $(1 / \Gamma(1+\alpha)) \int_{0}^{\infty}|f(x)|(d x)^{\alpha}<k<\infty$. The Yang-Laplace transforms of $f(x)$ is given by $[18,22]$

$$
\begin{aligned}
& L_{\alpha}\{f(x)\}= f_{s}^{L, \alpha}(s) \\
&:=\frac{1}{\Gamma(1+\alpha)} \int_{0}^{\infty} E_{\alpha}\left(-s^{\alpha} x^{\alpha}\right) f(x)(d x)^{\alpha}, \\
& 0<\alpha \leq 1,
\end{aligned}
$$

where the latter integral converges and $s^{\alpha} \in R^{\alpha}$.

Definition 7. The inverse formula of the Yang-Laplace transforms of $f(x)$ is given by $[18,22]$ :

$$
\begin{aligned}
L_{\alpha}^{-1}\left\{f_{s}^{L, \alpha}(s)\right\}= & f(t) \\
& :=\frac{1}{(2 \pi)^{\alpha}} \int_{\beta-i \infty}^{\beta+i \infty} E_{\alpha}\left(s^{\alpha} t^{\alpha}\right) f_{s}^{L, \alpha}(s)(d s)^{\alpha}, \\
& 0<\alpha \leq 1,
\end{aligned}
$$

where $s^{\alpha}=\beta^{\alpha}+i^{\alpha} \omega^{\alpha}$; fractal imaginary unit $i^{\alpha}$ and $\operatorname{Re}(s)=$ $\beta>0$.

\section{Local Fractional Function Decomposition Method}

In this section we will present the local fractional function decomposition method.

At first, we present the local fractional differential equation

$$
\begin{gathered}
\frac{\partial^{2 \alpha} u(x, t)}{\partial t^{2 \alpha}}+k_{1} \frac{\partial^{\alpha} u(x, t)}{\partial t^{\alpha}}+k_{2} \frac{\partial^{2 \alpha} u(x, t)}{\partial x^{2 \alpha}} \\
+k_{3} \frac{\partial^{\alpha} u(x, t)}{\partial x^{\alpha}}=f(x, t)
\end{gathered}
$$

with constants $k_{1}, k_{2}, k_{3}, 0<\alpha \leq 1 / 2$ and with boundary and initial conditions

$$
\begin{gathered}
u(0, t)=u(l, t)=0, \\
u(x, 0)=\varphi(x), \\
\frac{\partial^{\alpha} u(x, o)}{\partial t^{\alpha}}=\psi(x) .
\end{gathered}
$$

Now we discuss the solution of (10). 
According to the decomposition of the local fractional function, with respect to the system $\left\{\sin _{\alpha} n^{\alpha}(\pi x / l)^{\alpha}\right\}$, the following functions coefficients can be given by

$$
\begin{gathered}
u(x, t)=\sum_{n=1}^{\infty} v_{n}(t) \sin _{\alpha} n^{\alpha}\left(\frac{\pi x}{l}\right)^{\alpha}, \\
f(x, t)=\sum_{n=1}^{\infty} f_{n}(t) \sin _{\alpha} n^{\alpha}\left(\frac{\pi x}{l}\right)^{\alpha}, \\
\varphi(x)=\sum_{n=1}^{\infty} C_{n} \sin _{\alpha} n^{\alpha}\left(\frac{\pi x}{l}\right)^{\alpha}, \\
\psi(x)=\sum_{n=1}^{\infty} D_{n} \sin _{\alpha} n^{\alpha}\left(\frac{\pi x}{l}\right)^{\alpha},
\end{gathered}
$$

where

$$
\begin{aligned}
f_{n}(t) & =\frac{2}{l^{\alpha}} \int_{0}^{l} f(x, t) \sin _{\alpha} n^{\alpha}\left(\frac{\pi x}{l}\right)^{\alpha}(d x)^{\alpha}, \\
C_{n} & =\frac{2}{l^{\alpha}} \int_{0}^{l} \varphi(x) \sin _{\alpha} n^{\alpha}\left(\frac{\pi x}{l}\right)^{\alpha}(d x)^{\alpha}, \\
D_{n} & =\frac{2}{l^{\alpha}} \int_{0}^{l} \varphi(x) \sin _{\alpha} n^{\alpha}\left(\frac{\pi x}{l}\right)^{\alpha}(d x)^{\alpha} .
\end{aligned}
$$

Substituting (12) into (10) implies that

$$
\begin{gathered}
\frac{\partial^{\alpha} v_{n}(t)}{\partial t^{\alpha}}+k_{1} \frac{\partial^{\alpha} v_{n}(t)}{\partial t^{\alpha}}+k_{2}\left(\frac{n \pi}{l}\right)^{2 \alpha} v_{n}(t) \\
+k_{3}\left(\frac{n \pi}{l}\right)^{\alpha} v_{n}(t)=f_{n}(t), \\
v_{n}(0)=C_{n}, \quad v_{n}^{\prime}(0)=D_{n} .
\end{gathered}
$$

Suppose that the Yang-Laplace transforms of functions $v_{n}(t)$ and $f_{n}(t)$ are $V_{n}(s)$ and $F_{n}(s)$, respectively. Then we obtain

$$
\begin{aligned}
& s^{2 \alpha} V_{n}(s)-C_{n} s^{\alpha}-D_{n}+k_{1}\left(s^{\alpha} V_{n}(s)-D_{n}\right) \\
& +k_{2}\left(\frac{n \pi}{l}\right)^{2 \alpha} V_{n}(s)+k_{3}\left(\frac{n \pi}{l}\right)^{\alpha} V_{n}(s)=F_{n}(s) .
\end{aligned}
$$

That is

$$
\begin{aligned}
V_{n}(s)= & \frac{D_{n}+k_{1} D_{n}+C_{n} s^{\alpha}}{s^{2 \alpha}+k_{1} s^{\alpha}+k_{2}(n \pi / l)^{2 \alpha}+k_{3}(n \pi / l)^{\alpha}} \\
& +\frac{F_{n}(s)}{s^{2 \alpha}+k_{1} s^{\alpha}+k_{2}(n \pi / l)^{2 \alpha}+k_{3}(n \pi / l)^{\alpha}} .
\end{aligned}
$$

Hence, we have

$$
\begin{aligned}
& v_{n}(t) \\
& =L_{\alpha}^{-1}\left[V_{n}(s)\right] \\
& =\frac{1}{(2 \pi)^{\alpha}} \int_{\beta-i \infty}^{\beta+i \infty} E_{\alpha}\left(s^{\alpha} t^{\alpha}\right) V_{n}(s)(d s)^{\alpha} \\
& =\frac{1}{(2 \pi)^{\alpha}} \int_{\beta-i \infty}^{\beta+i \infty} E_{\alpha}\left(s^{\alpha} t^{\alpha}\right) \\
& \quad \times \frac{F_{n}(s)}{s^{2 \alpha}+k_{1} s^{\alpha}+k_{2}(n \pi / l)^{2 \alpha}+k_{3}(n \pi / l)^{\alpha}}(d s)^{\alpha} \\
& +\frac{1}{(2 \pi)^{\alpha}} \int_{\beta-i \infty}^{\beta+i \infty} E_{\alpha}\left(s^{\alpha} t^{\alpha}\right) \\
& \quad \times \frac{D_{n}+k_{1} D_{n}+C_{n} s^{\alpha}}{s^{2 \alpha}+k_{1} s^{\alpha}+k_{2}(n \pi / l)^{2 \alpha}+k_{3}(n \pi / l)^{\alpha}}(d s)^{\alpha} .
\end{aligned}
$$

Let

$$
\begin{aligned}
& v_{n}(t)=v_{1, n}(t)+v_{2, n}(t), \\
& v_{1, n}(t) \\
& =\frac{1}{(2 \pi)^{\alpha}} \\
& \quad \times \int_{\beta-i \infty}^{\beta+i \infty} E_{\alpha}\left(s^{\alpha} t^{\alpha}\right) \\
& \quad \times \frac{F_{n}(s)}{s^{2 \alpha}+k_{1} s^{\alpha}+k_{2}(n \pi / l)^{2 \alpha}+k_{3}(n \pi / l)^{\alpha}}(d s)^{\alpha}, \\
& =\frac{1}{(2 \pi)^{\alpha}}(t) \quad \times \int_{\beta-i \infty}^{\beta+i \infty} \times \frac{E_{\alpha}\left(s^{\alpha} t^{\alpha}\right)}{\quad \times \frac{D_{n}+k_{1} D_{n}+C_{n} s^{\alpha}}{s^{2 \alpha}+k_{1} s^{\alpha}+k_{2}(n \pi / l)^{2 \alpha}+k_{3}(n \pi / l)^{\alpha}}(d s)^{\alpha} .}
\end{aligned}
$$

Hence, we get

$$
\begin{aligned}
& V_{1, n}(s)=\frac{F_{n}(s)}{s^{2 \alpha}+k_{1} s^{\alpha}+k_{2}(n \pi / l)^{2 \alpha}+k_{3}(n \pi / l)^{\alpha}}, \\
& V_{2, n}(s)=\frac{D_{n}+k_{1} D_{n}+C_{n} s^{\alpha}}{s^{2 \alpha}+k_{1} s^{\alpha}+k_{2}(n \pi / l)^{2 \alpha}+k_{3}(n \pi / l)^{\alpha}} .
\end{aligned}
$$

Then, making use of (8) and (9) and rearranging integration sequence, we have the following several formulas about $v_{1, n}(t)$ and $v_{2, n}(t)$. 
(I) Suppose that

$$
\begin{gathered}
-\frac{1}{4} k_{1}^{2}+k_{2}\left(\frac{n \pi}{l}\right)^{2 \alpha}+k_{3}\left(\frac{n \pi}{l}\right)^{\alpha}>0 \\
s^{2 \alpha}+k_{1} s^{\alpha}+k_{2}\left(\frac{n \pi}{l}\right)^{2 \alpha}+k_{3}\left(\frac{n \pi}{l}\right)^{\alpha}=\left(s^{\beta}+\frac{k_{1}}{2}\right)^{2}+D_{n}^{\prime 2},
\end{gathered}
$$

where $D_{n}^{\prime}=\sqrt{-(1 / 4) k_{1}^{2}+k_{2}(n \pi / l)^{2 \alpha}+k_{3}(n \pi / l)^{\alpha}}$.

Then, we get

$$
\begin{aligned}
v_{1, n}(t)= & \frac{1}{D_{n}^{\prime} \Gamma(1+\alpha)} \\
& \times \int_{0}^{t} E_{\alpha}\left(\frac{-k_{1} \tau^{\alpha}}{2^{\alpha}}\right) \sin _{\alpha} D_{n}^{\prime} \tau f_{n}(t-\tau)(d \tau)^{\alpha} .
\end{aligned}
$$

When

$$
V_{2, n}(s)=\frac{C_{n}\left(s^{\alpha}+\left(k_{1} / 2\right)\right)+\left(D_{n}+k_{1} D_{n}-\left(k_{1} / 2\right) C_{n}\right)}{\left(s^{\alpha}+\left(k_{1} / 2\right)\right)^{2}+D_{n}^{\prime 2}}
$$

we get

$$
\begin{aligned}
v_{2, n}(t)= & C_{n} E_{\alpha}\left(\frac{-k_{1} t^{\alpha}}{2^{\alpha}}\right) \cos _{\alpha} \sqrt{D^{\prime}} t^{\alpha} \\
& +\left(D_{n}+k_{1} D_{n}-\frac{k_{1}}{2} C_{n}\right) E_{\alpha}\left(\frac{-k_{1} t^{\alpha}}{2^{\alpha}}\right) \sin _{\alpha} \sqrt{D^{\prime}} t^{\alpha} .
\end{aligned}
$$

(II) If

$$
\begin{gathered}
-\frac{1}{4} k_{1}^{2}+k_{2}\left(\frac{n \pi}{l}\right)^{2 \alpha}+k_{3}\left(\frac{n \pi}{l}\right)^{\alpha}=0, \\
s^{2 \alpha}+k_{1} s^{\alpha}+k_{2}\left(\frac{n \pi}{l}\right)^{2 \alpha}+k_{3}\left(\frac{n \pi}{l}\right)^{\alpha}=\left(s^{\alpha}+\frac{k_{1}}{2}\right)^{2},
\end{gathered}
$$

then we have

$$
v_{1, n}(t)=\frac{1}{\Gamma(1+\beta)} \int_{0}^{t} E_{\alpha}\left(\frac{-k_{1} \tau^{\alpha}}{2^{\alpha}}\right) \tau^{\alpha} f_{n}(t-\tau)(d \tau)^{\alpha} .
$$

When

$$
V_{2, n}(s)=\frac{C_{n}\left(s^{\alpha}+\left(k_{1} / 2\right)\right)+\left(D_{n}+k_{1} D_{n}-\left(k_{1} / 2\right) C_{n}\right)}{\left(s^{\alpha}+\left(k_{1} / 2\right)\right)^{2}},
$$

we arrive at

$$
\begin{aligned}
v_{2, n}(t)= & \frac{C_{n}}{\Gamma(1+\alpha)} E_{\alpha}\left(\frac{-k_{1} t^{\alpha}}{2^{\alpha}}\right) \\
& +\frac{D_{n}+k_{1} D_{n}-\left(k_{1} / 2\right) C_{n}}{\Gamma(1+\alpha)} t^{\alpha} E_{\alpha}\left(\frac{-k_{1} t^{\alpha}}{2^{\alpha}}\right) .
\end{aligned}
$$

(III) Let

$$
\begin{gathered}
-\frac{1}{4} k_{1}^{2}+k_{2}\left(\frac{n \pi}{l}\right)^{2 \alpha}+k_{3}\left(\frac{n \pi}{l}\right)^{\alpha}<0, \\
s^{2 \alpha}+k_{1} s^{\alpha}+k_{2}\left(\frac{n \pi}{l}\right)^{2 \alpha}+k_{3}\left(\frac{n \pi}{l}\right)^{\alpha}=\left(s^{\alpha}+\frac{k_{1}}{2}\right)^{2}-D_{n}^{\prime 2},
\end{gathered}
$$

where $D_{n}^{\prime}=\sqrt{(1 / 4) k_{1}^{2}-k_{2}(n \pi / l)^{2 \alpha}-k_{3}(n \pi / l)^{\alpha}}$.

Then, we have

$$
\begin{aligned}
v_{1, n}(t)= & \frac{1}{\Gamma(1+\alpha) D_{n}^{\prime}} \\
& \times \int_{0}^{t} E_{\alpha}\left(\frac{-k_{1} \tau^{\alpha}}{2^{\alpha}}\right) s h_{\alpha} D_{n}^{\prime} \tau^{\alpha} f_{n}(t-\tau)(d \tau)^{\beta} .
\end{aligned}
$$

When

$$
\begin{aligned}
V_{2, n}(s) & \\
= & \frac{C_{n}\left(s^{\alpha}+\left(k_{1} / 2\right)\right)+\left(D_{n}+k_{1} D_{n}-\left(k_{1} / 2\right) C_{n}\right)}{\left(s^{\alpha}+\left(k_{1} / 2\right)\right)^{2}-D_{n}^{\prime 2}} \\
= & \frac{\left(k_{1}+1\right) D_{n}+\left(D_{n}^{\prime}-\left(k_{1} / 2\right)\right) C_{n}}{2 D_{n}^{\prime}\left(s^{\alpha}+\left(k_{1} / 2\right)-D_{n}^{\prime}\right)} \\
& +\frac{\left(D_{n}^{\prime}+\left(k_{1} / 2\right)\right) C_{n}-\left(k_{1}+1\right) D_{n}}{2 D_{n}^{\prime}\left(s^{\alpha}+\left(k_{1} / 2\right)+D_{n}^{\prime}\right)},
\end{aligned}
$$

we obtain

$$
\begin{aligned}
v_{2, n}(t)= & \frac{\left(k_{1}+1\right) D_{n}+\left(D_{n}^{\prime}-\left(k_{1} / 2\right)\right) C_{n}}{2 D_{n}^{\prime}} \\
& \times E_{\alpha}\left(\left(D_{n}^{\prime}-\frac{k_{1}}{2^{\alpha}}\right) t^{\alpha}\right) \\
& +\frac{\left(D_{n}^{\prime}+\left(k_{1} / 2\right)\right) C_{n}-\left(k_{1}+1\right) D_{n}}{2 D_{n}^{\prime}} \\
& \times E_{\alpha}\left(\left(-D_{n}^{\prime}-\frac{k_{1}}{2^{\alpha}}\right) t^{\alpha}\right) .
\end{aligned}
$$

The above results are the desired solutions.

\section{Illustrative Examples}

In order to illustrate the above results in Section 3, we give the following several examples.

Example 1. The local fractional Laplace differential equation is written in the following form $[18,19]$ :

$$
\frac{\partial^{2 \alpha} u(x, t)}{\partial x^{2 \alpha}}+\frac{\partial^{2 \alpha} u(x, t)}{\partial t^{2 \alpha}}=0
$$


subjected to the boundary and initial conditions described by

$$
\begin{gathered}
u(x, 0)=\sin _{\alpha}\left(x^{\alpha}\right), \\
\frac{\partial^{\alpha} u(x, o)}{\partial t^{\alpha}}=\sin _{\alpha}\left(x^{\alpha}\right), \\
u(0, t)=u(\pi, t)=0 .
\end{gathered}
$$

From (33), the final solution can be easily deduced as follows:

$$
u(x, t)=\sin _{\alpha}\left(x^{\alpha}\right) E_{\alpha}\left(t^{\alpha}\right) .
$$

Example 2. We consider the following inhomogeneous wave equation with local fractional derivative:

$$
\frac{\partial^{2 \alpha} u(x, t)}{\partial t^{2 \alpha}}-\frac{\partial^{2 \alpha} u(x, t)}{\partial x^{2 \alpha}}-\sin _{\alpha} x^{\alpha}=0
$$

subjected to the boundary and initial conditions

$$
\begin{gathered}
u(x, 0)=\sin _{\alpha}\left(x^{\alpha}\right), \\
\frac{\partial^{\alpha} u(x, o)}{\partial t^{\alpha}}=0, \\
u(0, t)=u(\pi, t)=0 .
\end{gathered}
$$

In order to find its solution, we suppose that

$$
\begin{gathered}
u(x, t)=\sum_{n=1}^{\infty} v_{n}(t) \sin _{\alpha} n^{\alpha} x^{\alpha}, \\
f(x, t)=\sin _{\alpha} x^{\alpha}=\sum_{n=1}^{\infty} f_{n}(t) \sin _{\alpha} n^{\alpha} x^{\alpha} \\
\varphi(x)=\sin _{\alpha} x^{\alpha}=\sum_{n=1}^{\infty} C_{n} \sin _{\alpha} n^{\alpha} x^{\alpha} \\
\psi(x)=0=\sum_{n=1}^{\infty} D_{n} \sin _{\alpha} n^{\alpha} x^{\alpha}
\end{gathered}
$$

which leads to

$$
\begin{gathered}
f_{n}(t)=0, \quad(n \neq 1), \\
f_{1}(t)=1, \\
C_{n}=0, \quad(n \neq 1), \\
C_{1}=1, \\
D_{n}=0 .
\end{gathered}
$$

Contrasting (37) with (35), we directly get

$$
\begin{gathered}
k_{1}=k_{3}=0, \\
k_{2}=-1, \\
D_{n}^{\prime}=\sqrt{\frac{1}{4} k_{1}^{2}-k_{2}\left(\frac{n \pi}{l}\right)^{2 \alpha}-k_{3}\left(\frac{n \pi}{l}\right)^{\alpha}}=1, \quad(n=1), \\
D_{n}^{\prime}=0, \quad(n \neq 1) .
\end{gathered}
$$

According to (30) and (32), we can derive

$$
v_{n}(t)=0, \quad(n \neq 1),
$$

$$
\begin{aligned}
& v_{1,1}(t) \\
& =\frac{1}{\Gamma(1+\alpha) D_{1}^{\prime}} \int_{0}^{t} E_{\alpha}\left(\frac{-k_{1} \tau^{\alpha}}{2^{\alpha}}\right) s h_{\alpha} D_{1}^{\prime} \tau^{\alpha} f_{1}(t-\tau)(d \tau)^{\alpha} \\
& =\frac{1}{\Gamma(1+\alpha)} \int_{0}^{t} s h_{\alpha} \tau^{\alpha}(d \tau)^{\alpha} \\
& =\frac{E_{\alpha}\left(t^{\alpha}\right)+E_{\alpha}\left(-t^{\alpha}\right)}{2}-1 \\
& v_{1,2}(t)=\frac{E_{\alpha}\left(t^{\alpha}\right)+E_{\alpha}\left(-t^{\alpha}\right)}{2}
\end{aligned}
$$

Conclusively, we get

$$
v_{1}(t)=E_{\alpha}\left(t^{\alpha}\right)+E_{\alpha}\left(-t^{\alpha}\right)-1, \quad v_{n}(t)=0, \quad(n \neq 1) .
$$

Thus, we obtain

$$
u(x, t)=\left[E_{\alpha}\left(t^{\alpha}\right)+E_{\alpha}\left(-t^{\alpha}\right)-1\right] \sin _{\alpha}\left(x^{\alpha}\right) .
$$

Example 3. The inhomogeneous wave equation with local fractional differential operator is written in the following form:

$$
\frac{\partial^{2 \alpha} u(x, t)}{\partial t^{2 \alpha}}+\frac{\partial^{2 \alpha} u(x, t)}{\partial x^{2 \alpha}}=1 .
$$

The boundary and initial conditions are described by

$$
\begin{gathered}
u(x, 0)=\sin _{\alpha} x^{\alpha}, \\
\frac{\partial^{\alpha} u(x, o)}{\partial t^{\alpha}}=0 .
\end{gathered}
$$

In order to find the solution of (46), we set

$$
\begin{gathered}
u(x, t)=\sum_{n=1}^{\infty} v_{n}(t) \sin _{\alpha} n^{\alpha} x^{\alpha}, \\
f(x, t)=1=\sum_{n=1}^{\infty} f_{n}(t) \sin _{\alpha} n^{\alpha} x^{\alpha}, \\
\varphi(x)=\sin _{\alpha} x^{\alpha}=\sum_{n=1}^{\infty} C_{n} \sin _{\alpha} n^{\alpha} x^{\alpha}, \\
\psi(x)=0=\sum_{n=1}^{\infty} D_{n} \sin _{\alpha} n^{\alpha} x^{\alpha} .
\end{gathered}
$$

Hence, we get

$$
\begin{gathered}
f_{n}(t)=\frac{2\left[1-(-1)^{n}\right]}{n}, \\
C_{n}=0 \quad(n \neq 1), \\
C_{1}=1, \\
D_{n}=0 .
\end{gathered}
$$


Let

$$
\begin{gathered}
s^{2 \alpha}+n^{2 \alpha}=\left(s^{\alpha}\right)^{2}+D_{n}^{\prime 2}, \\
D_{n}^{\prime}=n^{\alpha} .
\end{gathered}
$$

Making use of (21) and (23),we can write

$$
\begin{aligned}
v_{1, n}(t) & =\frac{1}{D_{n}^{\prime} \Gamma(1+\alpha)} \frac{2\left[1-(-1)^{n}\right]}{n^{\alpha}} \int_{0}^{t} \sin _{\alpha}(n \tau)^{\alpha}(d \tau)^{\alpha} \\
& =\left(1-\cos _{\alpha}(n \tau)^{\alpha}\right) \frac{2\left[1-(-1)^{n}\right]}{n^{\alpha}} .
\end{aligned}
$$

When

$$
V_{2, n}(t)=\frac{D_{n}+k_{1} D_{n}+C_{n} s^{\alpha}}{s^{2 \alpha}+k_{1} s^{\alpha}+k_{2}(n \pi / l)^{2 \alpha}+k_{3}(n \pi / l)^{\alpha}}=\frac{s^{\alpha}}{s^{2 \alpha}+n^{2 \alpha}},
$$

we obtain

$$
\begin{gathered}
v_{2,1}(t)=\cos _{\alpha} t^{\alpha}, \\
v_{2, n}(t)=0, \quad(n \neq 1) .
\end{gathered}
$$

Conclusively, we arrive at

$$
\begin{gathered}
v_{1}(t)=v_{1,1}(t)+v_{2,1}(t)=4-3 \cos _{\alpha} t^{\alpha}, \\
v_{n}(t)=\left(1-\cos _{\alpha}(n t)^{\alpha}\right) \frac{2\left[1-(-1)^{n}\right]}{n^{\alpha}}, \quad(n \neq 1) .
\end{gathered}
$$

Hence, we obtain the solution of (46) in the following form:

$$
\begin{aligned}
u(x, t)= & \left(4-3 \cos _{\alpha} t^{\alpha}\right) \sin _{\alpha}\left(\frac{\pi x}{l}\right)^{\alpha} \\
& +\sum_{n=2}^{\infty}\left(1-\cos _{\alpha}(n t)^{\alpha}\right) \frac{2\left[1-(-1)^{n}\right]}{n^{\alpha}} \sin _{\alpha} n^{\alpha}\left(\frac{\pi x}{l}\right)^{\alpha} .
\end{aligned}
$$

Example 4. The inhomogeneous wave equation with local fractional differential operator is written in the form

$$
\frac{\partial^{2 \alpha} u(x, t)}{\partial t^{2 \alpha}}-\frac{\partial^{2 \alpha} u(x, t)}{\partial x^{2 \alpha}}+\frac{\partial^{\alpha} u(x, t)}{\partial x^{\alpha}}-\cos _{\alpha} t^{\alpha} \cos _{\alpha} x^{\alpha}=0 .
$$

The boundary and initial conditions are presented as follows:

$$
\begin{gathered}
u(x, 0)=\cos _{\alpha}\left(x^{\alpha}\right), \\
\frac{\partial^{\alpha} u(x, o)}{\partial t^{\alpha}}=0, \\
u\left(-\frac{\pi}{2}, t\right)=u\left(\frac{\pi}{2}, t\right)=0 .
\end{gathered}
$$

Let

$$
\begin{gathered}
u(x, t)=\sum_{n=1}^{\infty} v_{n}(t) \cos _{\alpha} n^{\alpha} x^{\alpha}, \\
f(x, t)=\cos _{\alpha} t^{\alpha} \cos _{\alpha} x^{\alpha}=\sum_{n=1}^{\infty} f_{n}(t) \cos _{\alpha} n^{\alpha} x^{\alpha}, \\
\varphi(x)=\cos _{\alpha} x^{\alpha}=\sum_{n=1}^{\infty} C_{n} \cos _{\alpha} n^{\alpha} x^{\alpha} \\
\psi(x)=0=\sum_{n=1}^{\infty} D_{n} \cos _{\alpha} n^{\alpha} x^{\alpha} .
\end{gathered}
$$

We can write

$$
\begin{gathered}
f_{n}(t)=0, \quad(n \neq 1), \\
f_{1}(t)=\cos _{\alpha} t^{\alpha}, \\
C_{n}=0, \quad(n \neq 1), \\
C_{1}=0, \\
D_{n}=0 .
\end{gathered}
$$

Obviously, we have

$$
\begin{array}{cc}
k_{1}=0, & k_{2}=-1, \quad k_{3}=1, \\
D_{n}^{\prime}=0 .
\end{array}
$$

From (25) and (27) we obtain

$$
\begin{gathered}
v_{1,1}=v_{n}(t)=0, \quad(n \neq 1), \\
v_{1,1}(t)=\frac{1}{\Gamma(1+\alpha)} \int_{0}^{t} \cos _{\alpha}(t-\tau)^{\alpha}(d \tau)^{\alpha}=\sin _{\alpha} t^{\alpha} .
\end{gathered}
$$

Hence, the nondifferentiable solution of (56) reads as

$$
u(x, t)=\sin _{\alpha} t^{\alpha} \cos _{\alpha}\left(x^{\alpha}\right) .
$$

\section{Conclusions}

In this work we proposed the local fractional function decomposition method. The applications of the methods for solving the inhomogeneous wave equations with local fractional derivative are discussed in detail. The new technique is an efficient mathematical tool for the scientists to deal with local fractional differential equations.

\section{Conflict of Interests}

The authors declare that have no conflict of interests regarding this paper.

\section{Acknowledgments}

This work is supported by the National Natural Science Foundation of China (no. 609040410), the foundation and advanced technology research program of Henan province (112300410300), and the Nanyang Normal University (NYNU200749). 


\section{References}

[1] R. D. Driver, Ordinary and Delay Differential Equations, vol. 20 of Applied Mathematical Sciences, Springer, New York, NY, USA, 1977.

[2] I. Podlubny, Fractional differential equations, vol. 198 of Mathematics in Science and Engineering, Academic Press, San Diego, Calif, USA, 1999.

[3] A. A. Kilbas, H. M. Srivastava, and J. J. Trujillo, Theory and Applications of Fractional Differential Equations, vol. 204 of North-Holland Mathematics Studies, Elsevier Science, Amsterdam, The Netherlands, 2006.

[4] A. M. Wazwaz, Partial Differential Equations: Methods and Applications, Balkema, Rotterdam, The Netherlands, 2002.

[5] J. Fan and J. H. He, "Fractal derivative model for air permeability in hierarchic porous media," Abstract and Applied Analysis, vol. 2012, Article ID 354701, 7 pages, 2012.

[6] N. Heymans and I. Podlubny, "Physical interpretation of initial conditions for fractional differential equations with RiemannLiouville fractional derivatives," Rheologica Acta, vol. 45, no. 5, pp. 765-771, 2006.

[7] H. Karami, A. Babakhani, and D. Baleanu, "Existence results for a class of fractional differential equations with periodic boundary value conditions and with delay," Abstract and Applied Analysis, vol. 2013, Article ID 176180, 8 pages, 2013.

[8] D. Baleanu, K. Diethelm, E. Scalas, and J. J. Trujillo, Fractional Calculus Models and Numerical Methods, Series on Complexity, Nonlinearity and Chaos, World Scientific, Hackensack, NJ, USA, 2012.

[9] J. Hristov, "Heat-balance integral to fractional (half-time) heat diffusion sub-model," Thermal Science, vol. 14, no. 2, pp. 291-316, 2010.

[10] J. Hristov, "A short-distance integral-balance solution to a strong subdiffusion equation: a weak power-law profile," International Review of Chemical Engineering, vol. 2, no. 5, pp. 555563,2010 .

[11] H. Jafari and S. Seifi, "Homotopy analysis method for solving linear and nonlinear fractional diffusion-wave equation," Communications in Nonlinear Science and Numerical Simulation, vol. 14, no. 5, pp. 2006-2012, 2009.

[12] H. Jafari and H. Tajadodi, "He’s variational iteration method for solving fractional Riccati differential equation," International Journal of Differential Equations, vol. 2010, Article ID 764738, 8 pages, 2010.

[13] A. Atangana and A. Secer, "The time-fractional coupledKorteweg-de-Vries equations," Abstract and Applied Analysis, vol. 2013, Article ID 947986, 8 pages, 2013.

[14] A. Atangana and A. Kılıçman, "Analytical solutions of boundary values problem of $2 \mathrm{D}$ and $3 \mathrm{D}$ poisson and biharmonic equations by homotopy decomposition method," Abstract and Applied Analysis, vol. 2013, Article ID 380484, 9 pages, 2013.

[15] C. Li and Y. Wang, "Numerical algorithm based on Adomian decomposition for fractional differential equations," Computers and Mathematics with Applications, vol. 57, no. 10, pp. 1672-1681, 2009.

[16] V. Daftardar-Gejji and H. Jafari, "Adomian decomposition: a tool for solving a system of fractional differential equations," Journal of Mathematical Analysis and Applications, vol. 301, no. 2, pp. 508-518, 2005.

[17] C. Li, D. Qian, and Y. Chen, "On Riemann-Liouville and Caputo derivatives," Discrete Dynamics in Nature and Society, vol. 2011, Article ID 562494, 15 pages, 2011.
[18] X. J. Yang, Local Fractional Functional Analysis and Its Applications, Asian Academic Publisher, Hong Kong, 2011.

[19] X. J. Yang, Advanced Local Fractional Calculus and Its Applications, World Science, New York, NY, USA, 2012.

[20] X. J. Yang and D. Baleanu, "Fractal heat conduction problem solved by local fractional variation iteration method," Thermal Science, vol. 17, no. 2, pp. 625-628, 2013.

[21] A. M. Yang, Y. Z. Zhang, and Y. Long, "The Yang-Fourier transforms to heat-conduction in a semi-infinite fractal bar," Thermal Science, vol. 17, no. 3, pp. 707-713, 2013.

[22] C. F. Liu, S. S. Kong, and S. J. Yuan, "Reconstructive schemes for variational iteration method within Yang-Laplace transform with application to fractal heat conduction problem," Thermal Science, vol. 17, no. 3, pp. 715-721, 2013.

[23] Y.-J. Hao, H. M. Srivastava, H. Jafari, and X.-J. Yang, "Helmholtz and diffusion equations associated with local fractional derivative operators involving the Cantorian and Cantor-type cylindrical coordinates," Advances in Mathematical Physics, vol. 2013, Article ID 754248, 5 pages, 2013.

[24] X.-J. Yang, D. Baleanu, and J. A. Tenreiro Machado, "Systems of Navier-Stokes equations on Cantor sets," Mathematical Problems in Engineering, vol. 2013, Article ID 769724, 8 pages, 2013.

[25] M.-S. Hu, R. P. Agarwal, and X.-J. Yang, "Local fractional Fourier series with application to wave equation in fractal vibrating string," Abstract and Applied Analysis, vol. 2012, Article ID 567401, 15 pages, 2012.

[26] X. J. Yang, D. Baleanu, and W. P. Zhong, "Approximation solutions for diffusion equation on Cantor time-space," Proceeding of the Romanian Academy Series A, vol. 14, no. 2, pp. 127-133, 2013. 


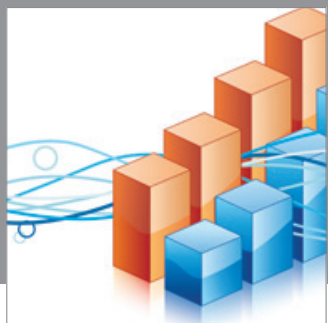

Advances in

Operations Research

mansans

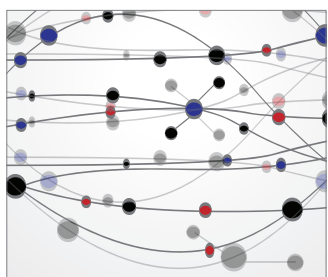

The Scientific World Journal
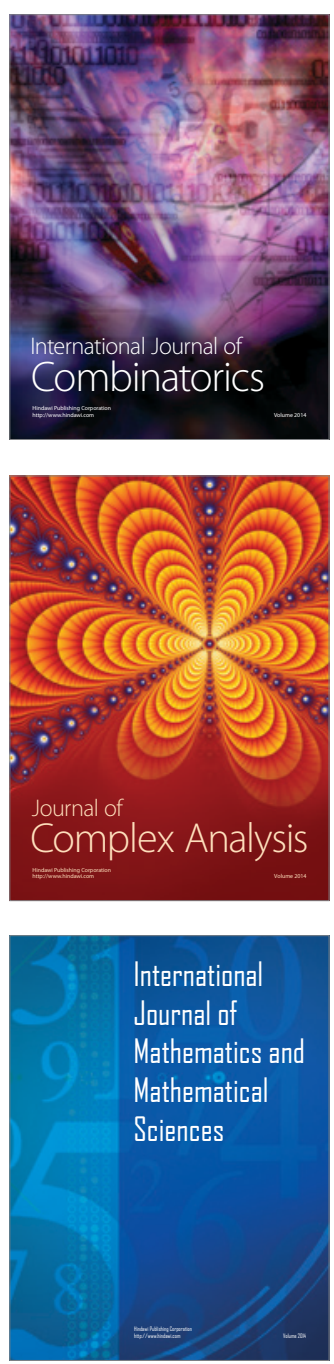
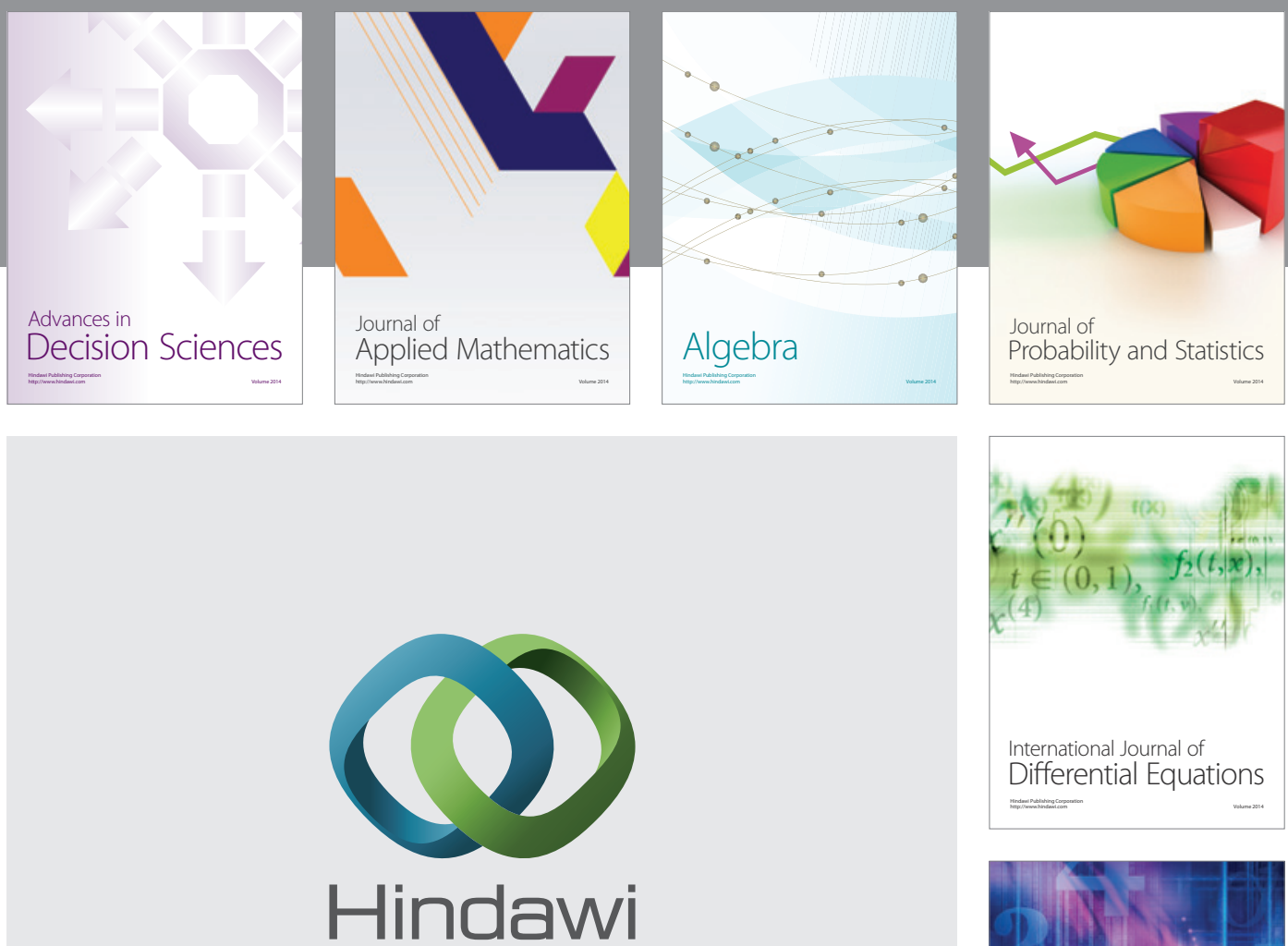

Submit your manuscripts at http://www.hindawi.com
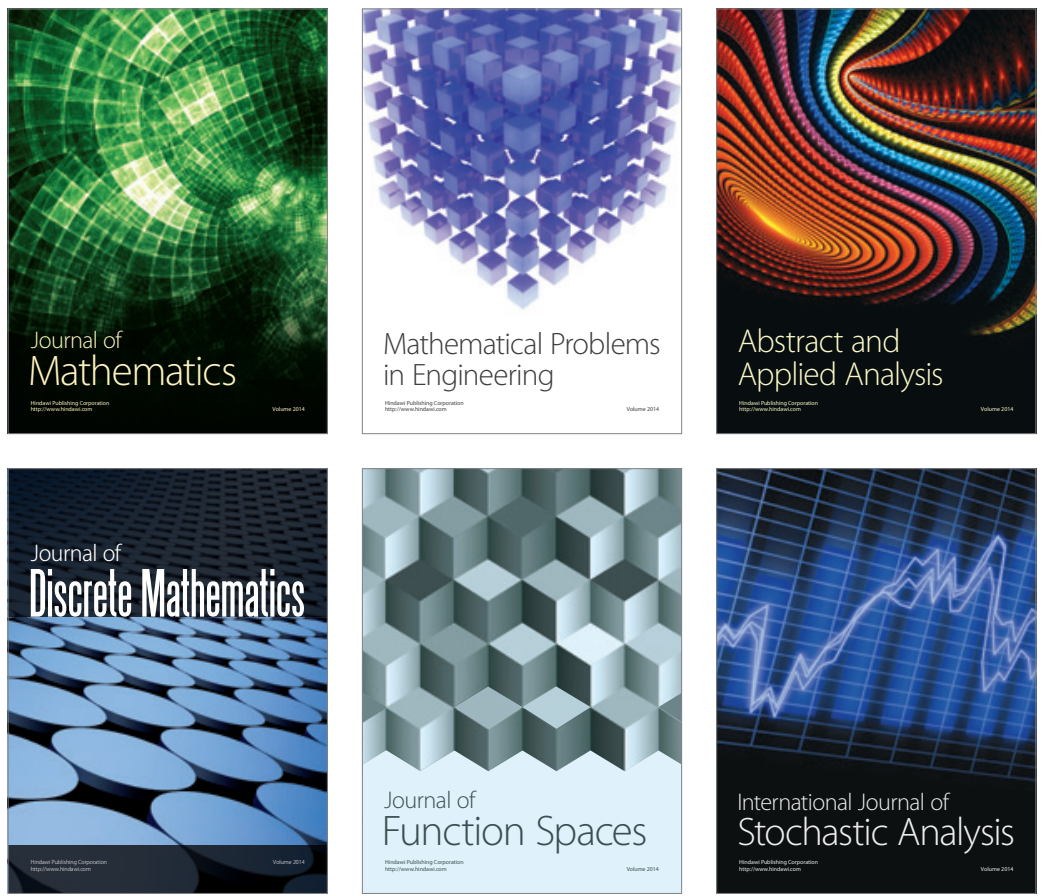

Journal of

Function Spaces

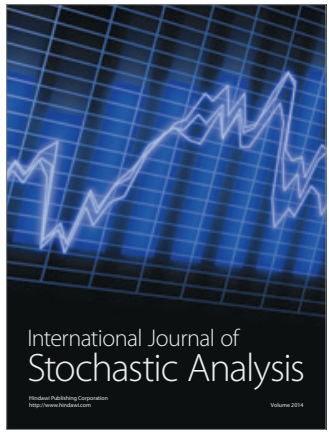

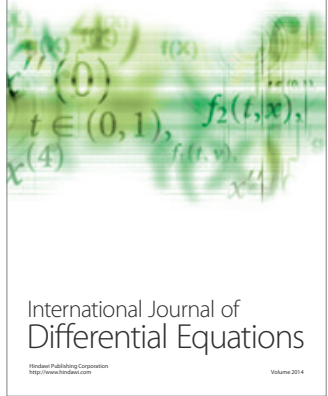
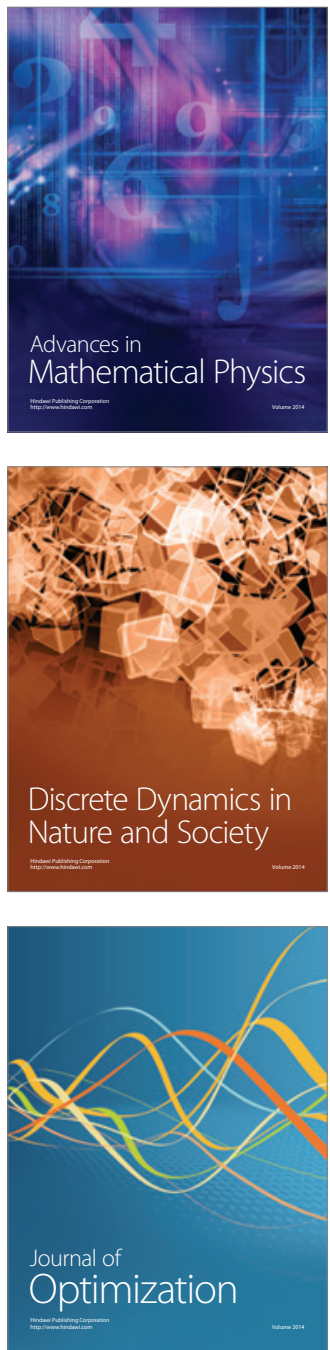